\title{
Investigation of intrinsic channel characteristics of hydrogenated amorphous silicon thin-film transistors by gated-four-probe structure
}

\author{
Chun-sung Chiang, Chun-ying Chen, ${ }^{\text {a) }}$ and Jerzy Kanicki ${ }^{\text {b) }}$ \\ Center for Display Technology and Manufacturing, Department of Electrical Engineering and Computer \\ Science, University of Michigan, Ann Arbor, Michigan 48109 \\ Kazushige Takechi \\ NEC Corp., Functional Devices Researh Laboratories, Kawasaki, Kanagawa, Japan
}

(Received 17 November 1997; accepted for publication 2 April 1998)

\begin{abstract}
We use a new hydrogenated amorphous silicon $(a-\mathrm{Si}: \mathrm{H})$ device structure, the gated-four-probe $a$-Si:H thin-film transistor (TFT), to investigate the intrinsic channel characteristics of inverted-staggered $a$-Si:H TFTs without the influence of source/drain series resistances. The experimental results have shown that, for the conventional $a$-Si:H TFT structure, the field-effect mobility, threshold voltage, and field-effect channel conductance activation energy have a strong dependence on $a$-Si:H thickness and TFT channel length. On the other hand, for the gated-four-probe $a$-Si:H TFT structure, these values are $a$-Si:H thickness and TFT channel length independent, clearly indicating that this new $a$-Si:H TFT structure can be effectively used to measure the channel intrinsic properties of $a-\mathrm{Si}: \mathrm{H}$ TFTs. (c) 1998 American Institute of Physics. [S0003-6951(98)04622-1]
\end{abstract}

Among the existing hydrogenated amorphous silicon ( $a$-Si:H) thin-film transistor (TFT) structures, the $n$-channel inverted-staggered TFT structure is the most popular in active-matrix liquid-crystal displays (AMLCDs) and image sensors. ${ }^{1}$ In this $n$-channel inverted-staggered TFT structure, the gate electrode is separated from the source and drain electrodes by a gate insulator (amorphous silicon nitride), an intrinsic $a-\mathrm{Si}: \mathrm{H}$, and a phosphorus-doped $(n+) a-\mathrm{Si}: \mathrm{H}$ layer. Under a positive above-threshold gate bias, an accumulation layer of electrons is induced to form a conducting channel near the $a$-Si:H/amorphous silicon nitride $\left(a-\mathrm{SiN}_{x}: \mathrm{H}\right)$ interface. Because of such inverted-staggered structure, the electrical performance of $a$-Si:H TFTs is determined by two factors: the intrinsic channel characteristics and parasitic series resistances. The characteristics of the intrinsic channel are mainly determined by the electronic quality of $a-\mathrm{Si}: \mathrm{H} / a-\mathrm{SiN}_{x}: \mathrm{H}$ interface, $a-\mathrm{Si}: \mathrm{H}$ bulk, and backchannel interface. On the other hand, the properties of parasitic resistances are affected by the quality of contacts between source/drain metal and $n+a-\mathrm{Si}: \mathrm{H}$, intrinsic $a-\mathrm{Si}: \mathrm{H}$ and $n+a$-Si:H film thickness, and gate-to-source/drain electrode overlap. The existence of parasitic series resistances makes it difficult to accurately determine $a-\mathrm{Si}: \mathrm{H}$ TFT intrinsic characteristics such as field-effect mobility $\left(\mu_{\mathrm{FE}}\right)$ and threshold voltage $\left(V_{T}\right)$ for optimized $a$-Si:H bulk material and $a-\mathrm{Si}: \mathrm{H} / a-\mathrm{SiN}_{x}: \mathrm{H}$ interface. To study the intrinsic performance of $a-\mathrm{Si}: \mathrm{H}$ TFTs, the effects of source/drain series resistances must be excluded.

We previously reported a new structure-the gated-fourprobe (GFP) $a$-Si:H TFT - to accurately measure the intrinsic characteristics of $a$-Si:H TFTs. ${ }^{2}$ In the GFP $a$-Si:H TFT structure, two additional narrow probes are placed between the source and drain electrodes of a conventional inverted-

\footnotetext{
a) Present address: Motorola Inc., Tempe, Arizona.

${ }^{b)}$ Corresponding author, electronic mail: kanicki@eecs.umich.edu
}

staggered $a$-Si:H TFT to sense the voltage difference along the conducting channel. By correlating this voltage difference with the source/drain current induced by the applied gate bias, the $a$-Si:H TFT intrinsic channel characteristics for electron conduction can be measured without the influence of source/drain series resistances. In a previous study, ${ }^{3}$ we employed a two-dimensional device simulator to predict the electrical characteristics of the new GFP $a$-Si:H TFT structure. The simulation results indicated that the effect of series resistances can be excluded in GFP $a$-Si:H TFTs, and determination of the intrinsic characteristics of $a-\mathrm{Si}: \mathrm{H}$ TFTs is possible with this new structure.

In this letter, we present experimental results for conventional inverted-staggered and GFP $a$-Si:H TFTs structures, which were fabricated at the same time on glass substrates (Corning 7059F). A $1500 \AA$ thick chromium (Cr) layer was first deposited by sputtering and patterned to form the gate electrode. Following the gate electrode formation, a $3000 \AA$ thick $a-\mathrm{SiN}_{x}: \mathrm{H}$ gate insulator, intrinsic $a$-Si:H channel layer (having thicknesses of 1500 and $3000 \AA$ ), and $500 \AA$ thick $n+a$-Si:H layer were deposited consecutively by plasmaenhanced chemical vapor deposition. A $2000 \AA$ thick $\mathrm{Cr}$ layer was then deposited by sputtering and patterned as source/drain electrodes and, for GFP TFTs, two additional narrow probes have been added. After the source/drain/probe patterning, a dry back-channel-etch process was used to remove the $n+a-\mathrm{Si}: \mathrm{H}$ using the patterned source/drain electrodes as the mask. To insure complete removal of $n+$ $a$-Si:H in the channel region, an over-etch process was used to etch off approximately $200 \AA$ of intrinsic $a$-Si:H layer within the channel.

An HP4156A semiconductor parameter analyzer was used to measure the current-voltage $(I-V)$ characteristics at different temperatures. For the $a-\mathrm{Si}: \mathrm{H}$ TFT, the gradual channel approximation equation in the linear region, $G$ $=I_{D} L / W V_{D}=\mu_{\mathrm{FE}} C_{i}\left(V_{\mathrm{GS}}-V_{T}\right)$, was used for $\mu_{\mathrm{FE}}$ and $V_{T}$ 


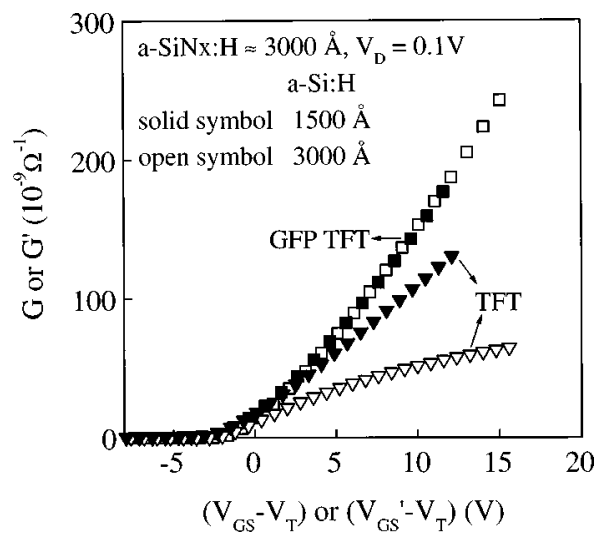

FIG. 1. Sheet conductance vs effective gate voltage characteristics for conventional $a$-Si:H TFT and GFP $a$-Si:H TFT structures having two different $a$-Si:H layer thicknesses. (W/L) for the conventional $a$-Si:H TFT and GFP $a$-Si:H TFT are (56/16) and (100/20), respectively. $V_{T}(1500 \AA) \approx 5.8 \mathrm{~V}$ and $V_{T}(3000 \AA) \approx 2.5 \mathrm{~V}$. Thickness of gate insulator $\left(a-\mathrm{SiN}_{x}: \mathrm{H}\right)$ is fixed at $3000 \AA$.

extraction, where $G$ is the normalized channel conductance, $C_{i}$ is the geometrical capacitance of the gate insulator, $V_{G}$ is the applied gate bias, and $W$ and $L$ are the channel width and length. For the GFP $a$-Si:H TFT structure, the device characteristics can be expressed as $G^{\prime}=I_{D} L^{\prime} / W\left(V_{B}-V_{A}\right)$ $=C_{i} \mu_{\mathrm{FE}}\left(V_{\mathrm{GS}}^{\prime}-V_{T}\right)$, where $G^{\prime}$ is the effective normalized channel conductance, $V_{A}$ and $V_{B}$ are the electrical potential for the two inner probes, $V_{\mathrm{GS}}^{\prime}=V_{\mathrm{GS}}-\left(V_{B}+V_{A}\right) / 2$ is the effective gate bias, and $L^{\prime}=\left(X_{B}-X_{A}\right)$ is the effective channel length. Since probes A and B only sense the electrical potential, $V_{A}$ and $V_{B}$ represent the true channel electrical potential. Hence, by using the GFP $a$-Si:H TFT structure, intrinsic field-effect mobility and intrinsic threshold voltage can be extracted from this equation without the influence of source/ drain series resistances. The field-effect channel conductance activation energy $\left(E_{A}\right)$ at different gate voltages was obtained from the slope of the Arrhenius $\ln (G)$ vs $T^{-1}$ plot, as $G \approx G_{0} \exp \left(-E_{A} / k T\right)$, where $G_{0}$ is a constant, $k$ is the Boltzmann constant, and $T$ is the absolute temperature.

Figure 1 shows the $G-V_{\mathrm{GS}}$ and $G^{\prime}-V_{\mathrm{GS}}^{\prime}$ characteristics in the linear region obtained for both $a$-Si:H TFT and GFP $a$-Si:H TFT structures having $a$-Si:H layers 1500 and 3000 $\AA$ thick. By fitting the experimental data to the above equations, we obtained for GFP $a$-Si:H TFTs the intrinsic fieldeffect mobility of about $0.85 \mathrm{~cm}^{2} / \mathrm{V} \mathrm{s}$. An effective gate bias, $V_{\mathrm{GS}}-V_{T}$ or $V_{\mathrm{GS}}^{\prime}-V_{T}$, was used in Fig. 1 to offset the effect of back-interface defect states on the threshold voltage; $V_{T}$ is 2.5 and $6 \mathrm{~V}$ for TFTs with 3000 and $1500 \AA a$-Si:H films, respectively. As can be seen in Fig. 1, a thicker $a$-Si:H layer (3000 $\AA$ ) causes a stronger reduction in the source-drain conductance of $a$-Si:H TFTs at higher gate voltages, indicating that a thicker $a$-Si:H layer introduces a higher device series resistance. However, for GFP $a$-Si:H TFTs, the $G^{\prime}-\left(V_{\mathrm{GS}}^{\prime}\right.$ $-V_{T}$ ) characteristics are nominally independent of $a$-Si:H film thickness, indicating that the effect of TFT series resistances has been excluded in this structure, which is consistent with the simulated data. ${ }^{3}$

The $G-V_{\mathrm{GS}}$ and $G^{\prime}-V_{\mathrm{GS}}^{\prime}$ characteristics for $a-\mathrm{Si}: \mathrm{H}$ TFTs and GFP $a$-Si:H TFTs having different channel lengths $\left(L\right.$ or $\left.L^{\prime}\right)$ are shown in Fig. 2. For $a$-Si:H TFTs in the linear region, a lower conductance is observed for a shorter channel

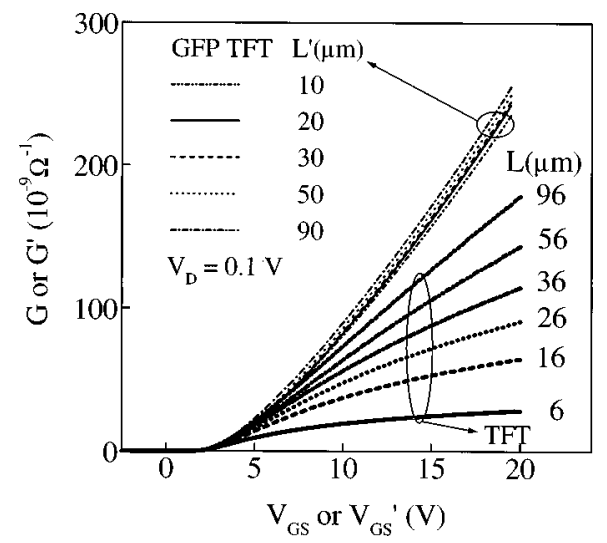

FIG. 2. Sheet conductance vs effective gate voltage characteristics for conventional $a$-Si:H TFT and GFP $a$-Si:H TFT structures having different channel lengths. $a$-Si:H and $a$ - $\mathrm{SiN}_{x}: \mathrm{H}$ films are both about $3000 \AA$ thick.

length TFT. Again, this is due to the effect of source/drain series resistances, which becomes stronger in $a$-Si:H TFTs with shorter channel lengths. ${ }^{4}$ For GFP $a$-Si:H TFTs, no substantial channel length dependence is observed in the $G^{\prime}$ $-V_{\mathrm{GS}}^{\prime}$ characteristics. Figure 3 shows the evolution of the field-effect mobilities and threshold voltages, extracted from Fig. 2, with channel lengths for both conventional $a-\mathrm{Si}: \mathrm{H}$ TFTs and GFP $a$-Si:H TFTs. As predicted by the numerical simulation, ${ }^{3}$ no dependence on channel length is observed for GFP $a$-Si:H TFTs. For $a$-Si:H TFTs, a strong dependence on channel length is observed, where longer channel length devices have higher extracted field-effect mobilities. This result clearly illustrates that the GFP $a$-Si:H TFT structure can effectively eliminate the effect of series resistances and provide the intrinsic device properties.

The field-effect channel conductance activation energy $\left(E_{A}\right)$ is associated with the position of the Fermi level $\left(E_{F}\right)$ in $a-\mathrm{Si}: \mathrm{H}$ that can be changed by gate biases. Its evolution with $V_{\mathrm{GS}}$ was used to calculate the density of states in $a-\mathrm{Si}: \mathrm{H}$ TFTs. ${ }^{5}$ Therefore, it is critical to know the exact $E_{A}$ values at different gate biases. As shown above, the series resistance has a significant effect on the overall source-to-drain conduction characteristics, especially for shorter channel length TFTs. It is expected, therefore, that series resistances will influence the determination of $E_{A}$ values, and the extent of that influence depends on the $a$-Si:H film thickness and the channel length. ${ }^{6}$ Figure 4 shows the evolution of $E_{A}$ with

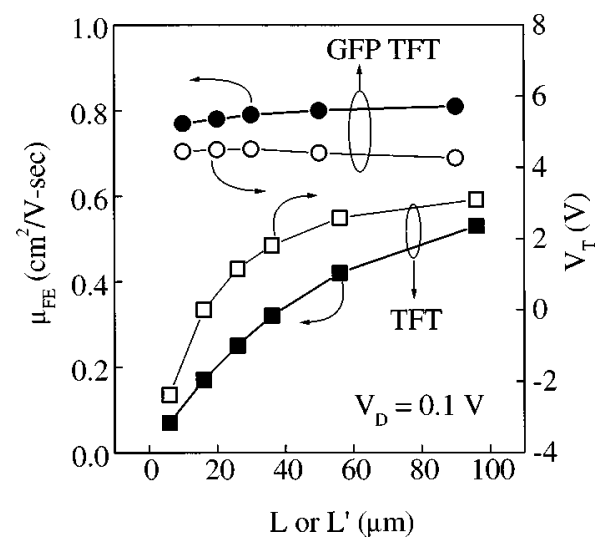

FIG. 3. Evolution of the field-effect mobility and threshold voltage as a function of channel length that were extracted from Fig. 2. 


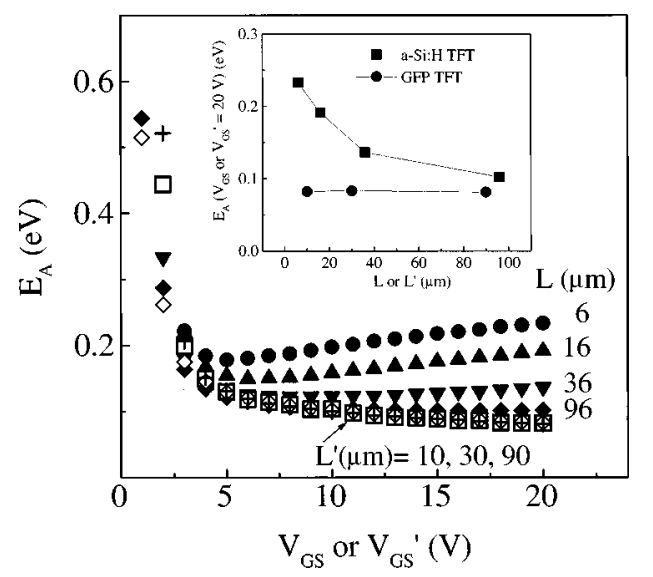

FIG. 4. Evolution of the channel conductance activation energy as a function of gate voltages for conventional $a$-Si:H TFT and GFP $a$-Si:H TFT structures. The channel length for $a$-Si:H TFTs is $6 \mu \mathrm{m}(\bullet), 16 \mu \mathrm{m}(\mathbf{\Delta}), 36$ $\mu \mathrm{m}(\mathbf{\nabla})$, and $96 \mu \mathrm{m}(\diamond)$; channel length for GFP TFTs is $10 \mu \mathrm{m}(\square), 30$ $\mu \mathrm{m}(\diamond)$, and $96 \mu \mathrm{m}(+)$. Channel widths for $a$-Si:H TFT and GFP TFT are 60 and $100 \mu \mathrm{m}$, respectively. Thickness of $a$-Si:H layer is $3000 \AA . E_{A}$ at $V_{\mathrm{GS}}\left(\right.$ or $\left.V_{\mathrm{GS}}^{\prime}\right)=20 \mathrm{~V}$ as a function of channel length is shown in the inset.

$V_{\mathrm{GS}}$ obtained for $a$-Si:H TFT and GFP $a$-Si:H TFT structures, where $a$-Si:H thickness is $3000 \AA$. At a higher gate bias $\left(V_{\mathrm{GS}}=20 \mathrm{~V}\right)$, the extracted $E_{A}$ values are $0.23,0.19$, 0.14 , and $0.10 \mathrm{eV}$ for $a$-Si:H TFTs with channel lengths of 6 , 16,36 , and $96 \mu \mathrm{m}$, respectively. For $V_{\mathrm{GS}}$ above the threshold voltage, $E_{A}$ increases with increasing $V_{\mathrm{GS}}$ for a short channel length TFT ( $L=6 \mu \mathrm{m})$, while it saturates at higher $V_{\mathrm{GS}}$ for a longer channel TFT $(L=96 \mu \mathrm{m})$. This channel-length dependence of $E_{A}$ is mainly due to the increasing influence of series resistances on TFT conduction characteristics with decreasing channel length and increasing gate voltage; variation of $E_{A}$ with $L$ at $V_{\mathrm{GS}}=20 \mathrm{~V}$ is shown in the inset of Fig.
4. On the other hand, for a GFP $a$-Si:H TFT with different channel lengths $(10,30$, and $90 \mu \mathrm{m})$, the $E_{A}$ value decreases with increasing effective gate bias, which indicates that $E_{F}$ approaches the conduction band-edge as gate-bias increases. In addition, $E_{A}$ values extracted for GFP TFTs do not depend on channel length (see inset of Fig. 4). This observation is consistent with theoretical calculation, ${ }^{3}$ since the effect of source/drain series resistances have been excluded in this structure. Hence, the $E_{A}$ value obtained for GFP $a-\mathrm{Si}: \mathrm{H}$ TFTs represents the true intrinsic $E_{A}$ value that can be used to characterize the quality of $a-\mathrm{Si}: \mathrm{H}$ TFT channel (including the $a-\mathrm{Si}: \mathrm{H} / a-\mathrm{SiN}_{x}: \mathrm{H}$ interface).

In this letter we have shown the experimental results for GFP $a$-Si:H TFTs that confirm our previous numerical simulation $^{3}$ indicating that GFP $a-\mathrm{Si}: \mathrm{H}$ TFTs are immune to the influence of source/drain series resistances. This work clearly demonstrates that the GFP $a$-Si:H TFT structure is a very useful tool for optimization and control of the intrinsic performance of $a$-Si:H TFTs during AMLCD fabrication.

The authors would like to thank the Center for Display Technology and Manufacturing at the University of Michigan for its financial support and the NEC Functional Devices Research Laboratories for its assistant in PECVD film deposition. The GFP $a$-Si:H TFT structure was developed and fabricated at the University of Michigan.

${ }^{1}$ M. J. Powell, IEEE Trans. Electron Devices 36, 2915 (1989).

${ }^{2}$ C.-Y. Chen and J. Kanicki, IEEE Electron Device Lett. 18, 340 (1997).

${ }^{3}$ C.-Y. Chen and J. Kanicki, SPIE Proc. 3014, 30 (1997).

${ }^{4}$ J. Kanicki, F. R. Libsch, J. Griffith, and R. Polastre, J. Appl. Phys. 69, 2339 (1991).

${ }^{5}$ R. E. I. Schropp, J. Snijder, and J. F. Verwey, J. Appl. Phys. 60, 643 (1986).

${ }^{6}$ C.-Y. Chen and J. Kanicki, Solid-State Electron. 42, 705 (1998). 